\title{
Effect of bootstrap current on the onset of neoclassical tearing mode
}

\author{
Q. Yu \\ Max-Planck-Institut für Plasmaphysik, 85748 Garching, Germany
}

\begin{abstract}
The effect of bootstrap current perturbation on the onset of neoclassical tearing modes (NTMs) is studied numerically based on two-fluid equations, using externally applied resonant magnetic perturbations (RMPs) as triggers. With increasing the bootstrap current density, a larger RMP is found to be required to excite the NTM's onset when the bi-normal electron fluid velocity is in the ion drift direction, being contrary to the conventional understanding that the bootstrap current perturbation is always destabilizing. For the electron fluid velocity in the electron drift direction, however, the opposite results are found.
\end{abstract}

*e-mail: qingquan.yu@ipp.mpg.de

Key words: neoclassical tearing mode, resonant magnetic perturbation, error field, plasma current density, magnetic island, two-fluid equations. 


\section{Introduction}

The onset of neoclassical tearing modes (NTMs) in tokamak experiments is often a type of forced magnetic reconnection caused by other MHD instabilities such as sawteeth, which provide the required resonant magnetic perturbation (RMP) via mode coupling to generate a sufficiently large seed magnetic island for the mode to grow [1-11]. NTMs are of particular importance for fusion plasmas, found to degrade tokamak plasma confinement or even cause plasma major disruption [311]. Understanding of the onset of NTMs remains to be important for a fusion reactor.

Existing theories for NTMs' onset are based on the modified Rutherford equation, including the tearing mode stability index $\Delta^{\prime}$, the curvature (Glasser) effect, and the contributions from the bootstrap current density perturbation and ion polarization current [1-3,12-20]. Except for the bootstrap current density perturbation, which is considered to be always destabilizing for a positive magnetic shear, all other effects are considered to be stabilizing for the mode onset, despite that the effect of the ion polarization current depends on the magnetic island rotation frequency and heat transport [12-17,21]. The error field and RMPs are also considered to be destabilizing based on single fluid results [2], providing seed islands for NTMs' growth if they are sufficiently large, similar to the triggering of NTMs by other instabilities.

In this paper the onset of the $\mathrm{m} / \mathrm{n}=3 / 2$ NTM driven by RMPs is studied numerically based on two-fluid equations [22-24], where $\mathrm{m} / \mathrm{n}$ is the poloidal/toroidal mode number. It is found that due to the change of the local electron density gradient around the resonant surface by RMPs, the local radial profile of the $\mathrm{m} / \mathrm{n}=0 / 0$ component of the bootstrap and plasma current density is significantly changed. As a result, when the bi-normal electron fluid velocity is in the ion drift direction, a larger RMP (or seed island) is required to trigger the NTM's onset for a larger bootstrap current density, being contrary to the conventional understanding that the bootstrap current perturbation is always destabilizing for the NTM growth [1-3]. For the bi-normal electron fluid velocity in the electron drift direction, however, the opposite result is found.

\section{Numerical model}

The four-field equations, the continuity equation, the generalized Ohm's law, the plasma vorticity equation, and the equation of motion in the parallel (to magnetic field) direction, are utilized [22]. Normalizing the length to the plasma minor radius a, the time $t$ to the resistive time $\tau_{R}=a^{2} \mu_{0} / \eta$ ( $\eta$ the plasma resistivity), the helical magnetic flux $\psi$ to $\mathrm{aB}_{0 \mathrm{t}}\left(\mathrm{B}_{0 \mathrm{t}}\right.$ is the equilibrium toroidal field), and the electron density $n_{\mathrm{e}}$ to its value at the magnetic axis, these equations become $[23,24]$

$$
\frac{d n_{e}}{d t}=d_{1} \nabla_{\|} j-\nabla_{\|}\left(n_{e} v_{\|}\right)+\nabla_{\perp}\left(D_{\perp} \nabla_{\perp} n_{e}\right)+S_{n}
$$




$$
\begin{aligned}
& \frac{d \psi}{d t}=E_{0}-\eta_{N}\left(j-j_{b}\right)-\frac{\eta_{N}}{v_{e i}} \frac{d j}{d t}+\Omega\left(\frac{T_{e}}{n_{e}} \nabla_{||} n_{e}+\nabla_{\|} T_{e}\right), \\
& \frac{d U}{d t}=S^{2} \nabla_{\|} j+\mu \nabla_{\perp}^{2} U+S_{m}, \\
& \frac{d v_{\|}}{d t}=-C_{S}^{2} \nabla_{\|} P / n_{e}+\mu \nabla_{\perp}^{2} v_{\|},
\end{aligned}
$$

using the large aspect ratio approximation and neglecting toroidal mode coupling, where

$$
\begin{aligned}
& \mathrm{d} / \mathrm{dt}=\partial / \partial \mathrm{t}+\mathbf{v}_{\perp} \cdot \nabla_{\perp}, \\
& \mathrm{j}=-\nabla_{\perp}^{2} \psi-2 \mathrm{nB}_{0 \mathrm{t}} /(\mathrm{mR})
\end{aligned}
$$

is the parallel plasma current density,

$$
\mathrm{U}=-\nabla_{\perp}^{2} \phi
$$

is the plasma vorticity, and $\phi$ is the stream function. The magnetic field is defined as $\mathbf{B}=\mathrm{B}_{0 t} \mathbf{e}_{t^{-}}$ $\left(\mathrm{k}_{\mathrm{t}} / \mathrm{k}_{\theta}\right) \mathrm{B}_{0 \mathrm{t}} \mathbf{e}_{\theta}+\nabla \psi \times \mathbf{e}_{\mathrm{t}}$, where $\mathrm{k}_{\theta}=\mathrm{m} / \mathrm{r}, \mathrm{k}_{\mathrm{t}}=\mathrm{n} / \mathrm{R}, \mathbf{e}_{\theta}$ and $\mathbf{e}_{\mathrm{t}}$ are the poloidal and toroidal unit vectors, and $\mathrm{r}$ and $\mathrm{R}$ are the minor and the major radius. The ion velocity $\mathbf{v}=\mathbf{v}_{\|}+\mathbf{v}_{\perp}$, including both the parallel and perpendicular component, and $\mathbf{v} \perp=\nabla \phi \times \mathbf{e}_{t} . \mathrm{P}=\mathrm{P}_{\mathrm{e}}=\mathrm{n}_{\mathrm{e}} \mathrm{T}_{\mathrm{e}}$ is the electron pressure, $\eta_{\mathrm{N}}=1$ the normalized resistivity. The bootstrap current density $\mathrm{j}_{\mathrm{b}}=-\mathrm{c}_{\mathrm{b}} \sqrt{ }_{\varepsilon}\left(\mathrm{c}_{\mathrm{T}} \mathrm{n}_{\mathrm{e}} T_{e}^{\prime}+\mathrm{T}_{\mathrm{e}} n_{e}^{\prime}\right) / \mathrm{B}_{\mathrm{p}}$, where $\mathrm{c}_{\mathrm{b}}$ is a constant of the order of unity, $\varepsilon=r / R, c_{T}=0.367, B_{p}$ the poloidal magnetic field, and the prime is for $\partial / \partial \mathrm{r}$. $\mathrm{E}_{0}$ is the equilibrium electric field, $S_{n}$ the particle source, and $S_{m}$ the poloidal momentum source leading to an equilibrium poloidal plasma rotation. $\Omega=\beta \mathrm{d}_{1}, \mathrm{~d}_{1}=\omega_{\mathrm{ce}} / v_{\mathrm{ei}}, \beta=4 \pi \mathrm{P}_{\mathrm{e}} / \mathrm{B}_{0 \mathrm{t}}^{2}, \omega_{\mathrm{ce}}$ and $v_{\mathrm{ei}}$ are the electron cyclotron and the collisional frequency, $S=\tau_{\mathrm{R}} / \tau_{\mathrm{A}}$, where $\tau_{\mathrm{A}}=\mathrm{a} / \mathrm{V}_{\mathrm{A}}$ is the toroidal Alfven time. $\mathrm{C}_{\mathrm{s}}, \mu$ and $\mathrm{D}_{\perp}$ are the normalized ion sound velocity, plasma viscosity, and perpendicular particle diffusivity. The cold ion assumption is made. The third term on the right hand side of equation (2), being proportional to $1 / v_{\mathrm{ei}}$, takes into account the electron inertia. The electron temperature $\mathrm{T}_{\mathrm{e}}$ is assumed to be a constant in above equations, and this assumption will be removed later in the last part of Section 3.

Equations (1)-(7) are solved simultaneously using the initial value code TM1 [23,24]. The effect of the $\mathrm{m} / \mathrm{n}=3 / 2 \mathrm{RMP}$ is taken into account by the boundary condition

$$
\left.\psi_{3 / 2}\right|_{\mathrm{r}=\mathrm{a}}=\psi_{\mathrm{a}, 3 / 2} \mathrm{aB}_{0 \mathrm{t}} \cos (3 \theta+2 \phi),
$$

where $\theta$ and $\phi$ are the poloidal and toroidal angle, and $\psi_{\mathrm{a}, 3 / 2}$ is the normalized amplitude of the $3 / 2$ component helical flux at $\mathrm{r}=\mathrm{a}$.

\section{Numerical results}

The radial profile of the original equilibrium plasma current density is of the form $\mathrm{j}_{0} \sim[1$ $\left.(\mathrm{r} / \mathrm{a})^{2}\right]^{4}$, leading to a monotonically increasing profile of the safety factor $\mathrm{q}$ with the $\mathrm{q}=3 / 2$ surface located at $r_{s}=0.611 a$. 
The input parameters are based on ASDEX Upgrade experimental parameters: $\mathrm{B}_{0 \mathrm{t}}=2 \mathrm{~T}, \mathrm{a}=0.5 \mathrm{~m}$, $\mathrm{R}=1.7 \mathrm{~m}, \mathrm{~T}_{\mathrm{e}}=2 \mathrm{keV}$, and $\mathrm{n}_{\mathrm{e}}=3 \times 10^{19} \mathrm{~m}^{-3}$, leading to $\mathrm{S}=2.6 \times 10^{8}, \mathrm{C}_{\mathrm{s}}=2 \times 10^{7}\left(\mathrm{a} / \tau_{\mathrm{R}}\right)$ and $\mathrm{d}_{1}=3.1 \times 10^{7} . \mathrm{A}$ parabolic profile for the equilibrium electron density is assumed, and the local equilibrium electron diamagnetic drift frequency is $\omega *_{0} 0=2.34 \times 10^{5} / \tau_{\mathrm{R}}\left(\mathrm{f}_{*_{\mathrm{e}} 0}=1.6 \mathrm{kHz}\right)$ at $\mathrm{r}_{\mathrm{s}}$ for $\mathrm{m}=3$ and $\Omega=2 \times 10^{4}$. The perpendicular plasma momentum and particle transport is assumed to be at an anomalous transport level of $\mu=0.2 \mathrm{~m}^{2} / \mathrm{s}=18.8\left(\mathrm{a}^{2} / \tau_{\mathrm{R}}\right)$ and $\mathrm{D}_{\perp}=\mu / 5$.

In tokamak experiments the core plasma rotation is essentially toroidal, while only the poloidal rotation is included in Eqs. (1)-(7) due to the large aspect ratio approximation. To guarantee a reasonable balance between the electromagnetic (EM) and viscous force, a larger plasma viscosity for the $\mathrm{m} / \mathrm{n}=0 / 0$ component, $\mu_{0 / 0}=18.8 \times 10^{2}$, is used in calculations based on the following considerations [25]: (a) The EM force in the toroidal direction is smaller by a factor $(\mathrm{n} / \mathrm{m})\left(\mathrm{r}_{\mathrm{s}} / \mathrm{R}\right)$ than that in the poloidal direction. (b) To have the same mode frequency due to the plasma rotation, the toroidal rotation velocity should be $(\mathrm{m} / \mathrm{n})\left(\mathrm{R} / \mathrm{r}_{\mathrm{s}}\right)$ times larger than the poloidal one. These two effects lead to a larger viscous force compared to the EM force for the toroidal rotation case by a factor $\left[(\mathrm{m} / \mathrm{n})\left(\mathrm{R} / \mathrm{r}_{\mathrm{s}}\right)\right]^{2}$, which is of the order $10^{2}$.

The normalized (to $\omega * \mathrm{e} 0$ ) local equilibrium electron fluid frequency at $\mathrm{r}_{\mathrm{s}}$ is defined as

$$
\omega_{\mathrm{n}} \equiv\left(1-\omega_{0}\right) \text {, }
$$

where $\omega_{0} \equiv-\omega_{\mathrm{E} 0} / \omega_{\mathrm{e} 0}$, and $\omega_{\mathrm{E} 0}$ the local equilibrium plasma rotation (the $\mathbf{E} \times \mathbf{B}$ rotation) frequency. A negative (positive) value of $\omega_{\mathrm{n}}\left(\omega_{0}\right)$ corresponds to a bi-normal electron fluid ( $\mathbf{E} \times \mathbf{B}$ rotation) velocity in the ion drift direction.

The time evolution of the normalized $\mathrm{m} / \mathrm{n}=3 / 2$ magnetic island width, calculated from $\mathrm{W}=4\left[\psi_{3 / 2} /\left(\mathrm{B}_{\mathrm{p}} \mathrm{q}^{\prime} / \mathrm{q}\right)\right]^{1 / 2}$ at $\mathrm{r}_{\mathrm{s}}$, is shown in figure 1 for $\omega_{\mathrm{n}}=-0.76$. When the equilibrium bootstrap current density fraction, $\mathrm{f}_{\mathrm{b}}$, is taken to be zero in calculations (red curves), the island width is less than 0.01a for $\psi_{\mathrm{a}, 3 / 2}=7 \times 10^{-5}$ (dashed) but grows to about $0.04 \mathrm{a}$ for a slightly large RMP, $\psi_{\mathrm{a}, 3 / 2}=8 \times 10^{-5}$ (solid), indicating the field penetration. Including the bootstrap current in calculations with $\mathrm{f}_{\mathrm{b}}=0.36$ at $\mathrm{r}_{\mathrm{s}}$ (black curves), the island width however remains at a low value about $0.01 \mathrm{a}$ up to $\psi_{\mathrm{a}, 3 / 2}=1.2 \times 10^{-4}$ (dashed), revealing the stabilizing role of the bootstrap current perturbation for the field penetration or the NTM's onset in this case. Slightly increasing the RMP amplitude to $\psi_{\mathrm{a}, 3 / 2}=1.3 \times 10^{-4}$ (solid), the island width grows to $0.13 \mathrm{a}$, being much larger than that for $\mathrm{f}_{\mathrm{b}}=0$ and showing the destabilizing role of the bootstrap current perturbation for a sufficiently large island, as expected from existing theories [1-3]. As a result, the local electron density profile is flattened, and the plasma rotation velocity is reduced to zero around the $q=3 / 2$ surface. Without applying the RMP, the $3 / 2$ mode is stable in the linear phase for $\mathrm{f}_{\mathrm{b}}=0$ to 0.36 . 


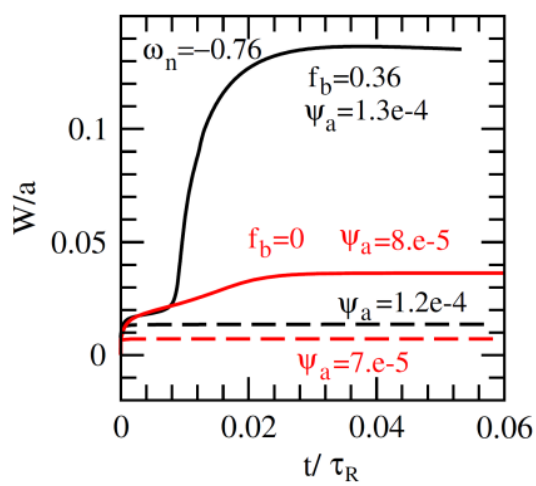

Figure $1 \quad$ Time evolution of the $m / n=3 / 2$ island width with $\omega_{n}=-0.76$ (the bi-normal electron fluid velocity in the ion drift direction). The red curves are for $f_{b}=0$, with $\psi_{a, 3 / 2}=7 \times 10^{-5}$ (dashed) and $8 \times 10^{-5}$ (solid). The black curves are for $f_{b}=036$, with $\psi_{a, 3 / 2}=1.2 \times 10^{-4}$ (dashed) and $1.3 \times 10^{-4}$ (solid). A larger RMP is required for field penetration for $f_{b}=0.36$ than that for $f_{b}=0$.

By scanning over the applied RMP amplitude in calculations with $\omega_{n}=-0.76$, the $m / n=3 / 2$ magnetic island width in steady state is shown as a function of $\psi_{\mathrm{a}, 3 / 2}$ in figure 2 for $\mathrm{f}_{\mathrm{b}}=0,0.18$ and 0.36. The different values of $\mathrm{f}_{\mathrm{b}}$ are obtained by using different values of $\mathrm{c}_{\mathrm{b}}$, so that other input parameters are unchanged (If using different equilibrium electron density profiles for different values of $f_{b}$, the diamagnetic drift frequency would be different). With $f_{b}=0$ (black curve), the island width jumps from a small value to about $0.04 \mathrm{a}$ at $\psi_{\mathrm{a}, 3 / 2}=8 \times 10^{-5}$ due to the field penetration. With $\mathrm{f}_{\mathrm{b}}=0.18$ (red), a larger RMP amplitude, $\psi_{\mathrm{a}, 3 / 2}=1.1 \times 10^{-4}$, is required for the penetration. Further increasing the local bootstrap current density fraction to $\mathrm{f}_{\mathrm{b}}=0.36$ (blue), the penetration threshold is even larger. This differs from existing theories that a larger bootstrap current density is more destabilizing for the NTM's onset or field penetration [1-3]. With increasing the bootstrap current density, the saturated island width is larger after the field penetration, as expected [1-3].

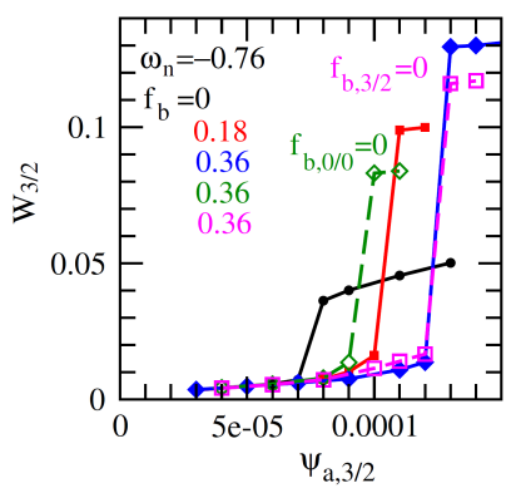

Figure $2 \quad$ The $m / n=3 / 2$ magnetic island width in steady state as a function of $\psi_{a, 3 / 2}$ for $f_{b}=0$ (black curve), 0.18 (red) and 0.36 (blue) at $r_{s}$ with $\omega_{n}=-0.76$. The penetration threshold is larger for a larger bootstrap current density fraction. The dashed green (magenta) curve is also for $f_{b}=0.36$, but the $m / n=0 / 0(3 / 2)$ component bootstrap current density is taken to be zero in calculations. 
In order to identify the underlying mechanism, calculations have also been carried out by taking the $\mathrm{m} / \mathrm{n}=0 / 0$ component of the bootstrap current density, $\mathrm{j}_{\mathrm{b}, 0 / 0}$, to be zero, while keeping other input parameters the same as those for the blue curve $\left(f_{b}=0.36\right)$, and the corresponding results are shown by the dashed green curve in figure 2 . In this case the penetration threshold is significantly reduced compared to that shown by the blue curve, revealing the stabilizing role due to the change of $\mathrm{j}_{\mathrm{b}, 0 / 0}$ from the equilibrium one by RMPs. When only the $3 / 2$ component bootstrap current density is take to be zero in calculations, keeping other input parameters unchanged, the penetration threshold remains the same (dashed magenta curve in figure 2).

By changing the momentum source in equation (3) to let the electron fluid velocity be in the electron drift direction with $\omega_{\mathrm{n}}=1.6$, the $\mathrm{m} / \mathrm{n}=3 / 2$ island width in steady state is shown as a function of $\psi_{\mathrm{a}, 3 / 2}$ in figure 3 for $\mathrm{f}_{\mathrm{b}}=0$ (black curve), 0.18 (red) and 0.36 (blue). The penetration threshold is however smaller for a larger bootstrap current density in this case, showing the destabilizing role of the bootstrap current perturbation. Taking the $m / n=0 / 0(3 / 2)$ component of the bootstrap current density to be zero in calculations, while keeping other input parameters the same as those for the blue curve, the results are shown by the dashed green (magenta) curve. In both cases the penetration threshold is significantly increased, showing the destabilizing roles of both the $0 / 0$ and the helical (3/2) component of the bootstrap current perturbations.

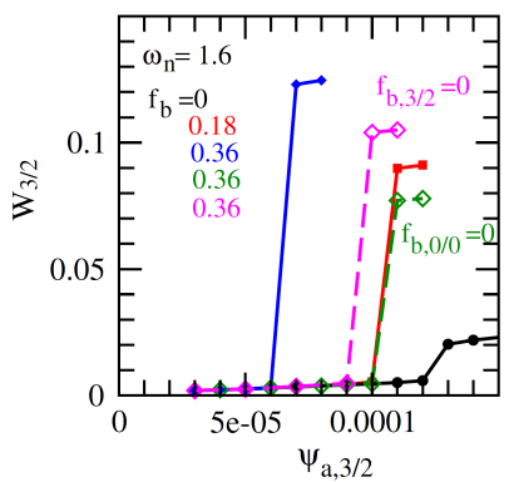

Figure 3 For the electron fluid velocity in the electron drift direction with $\omega_{n}=1.6$, the $\mathrm{m} / \mathrm{n}=3 / 2$ magnetic island width in steady state is shown as a function of $\psi_{a, 3 / 2}$ for $f_{b}=0$ (black curve), 0.18 (red) and 0.36 (blue) at $r_{s}$. The dashed green (magenta) curve is also for $f_{b}=0.36$, but the $m / n=0 / 0(3 / 2)$ component bootstrap current density is taken to be zero in calculations.

The relative change in the $\mathrm{m} / \mathrm{n}=0 / 0$ component of the bootstrap and plasma current density by RMPs can be defined as $\Delta \mathrm{j}_{\mathrm{b}, 0 / 0}=\left[\mathrm{j}_{\mathrm{b}, 0 / 0}(\mathrm{r})-\mathrm{j}_{\mathrm{b} 0}(\mathrm{r})\right] / \mathrm{j}_{0}\left(\mathrm{r}_{\mathrm{s}}\right)$ and $\Delta \mathrm{j}_{0 / 0}=\left[\mathrm{j}_{0 / 0}(\mathrm{r})-\mathrm{j}_{0}(\mathrm{r})\right] / \mathrm{j}_{0}\left(\mathrm{r}_{\mathrm{s}}\right)$, respectively, where $\mathrm{j}_{\mathrm{b}, 0 / 0}$ and $\mathrm{j}_{0 / 0}$ are the $0 / 0$ component of the bootstrap and plasma current density in nonlinear steady state, $\mathrm{j}_{\mathrm{b} 0}$ and $\mathrm{j}_{0}$ are the original equilibrium bootstrap and plasma current density. Local radial profiles 
of $\Delta \mathrm{j}_{0 / 0}$ (solid curve) and $\Delta \mathrm{j}_{\mathrm{b}, 0 / 0}$ (dashed) are shown in figure 4 by black curves for $\mathrm{f}_{\mathrm{b}}=0.18, \psi_{\mathrm{a}, 3 / 2}=$ $9 \times 10^{-5}$ and $\omega_{\mathrm{n}}=-0.76$. In this case the RMP has not penetrated in, and the island width is $0.01 \mathrm{a}$. The amplitude of $\Delta \mathrm{j}_{0 / 0}$ is about $7 \%$, largely corresponding to the change of the $0 / 0$ component bootstrap current density (the positive value of $\Delta \mathrm{j}_{\mathrm{b}, 0 / 0}$ will be explained later by equation (10)). The difference between them is due to the nonlinearity from the $\mathbf{v} \cdot \nabla \psi$ term, the parallel electron pressure gradient and electron inertia in Ohm's law, which act as an effective parallel electric field to change the $\mathrm{m} / \mathrm{n}=0 / 0$ component plasma current density [26]. More importantly, the radial $\Delta \mathrm{j}_{0 / 0}$ profile reveals that, compared to that of the original equilibrium one, the local radial gradient of $j_{0 / 0}$ is reversed on the inner side of the $\mathrm{q}=3 / 2$ surface but is decreased on the outer side (the absolute value of $j_{0 / 0}^{\prime} / j_{0 / 0}$ is increased since it is negative). This is similar to that caused by a local rf current drive at the resonant surface in the ion drift direction (co-current direction). Such a change in the local radial profile of $\mathrm{j}_{0} / 0$ by RMPs is stabilizing for the island growth, since it is known to decrease the $\Delta^{\prime}$ value. This explains the results shown in figures 1 and 2, i.e., the stabilizing effect of the bootstrap current perturbation on NTMs' onset for the electron fluid velocity in the ion drift direction. The amplitude of the $m / n=3 / 2$ component of the bootstrap current density perturbation is about two times smaller than that of the $0 / 0$ component in this case.

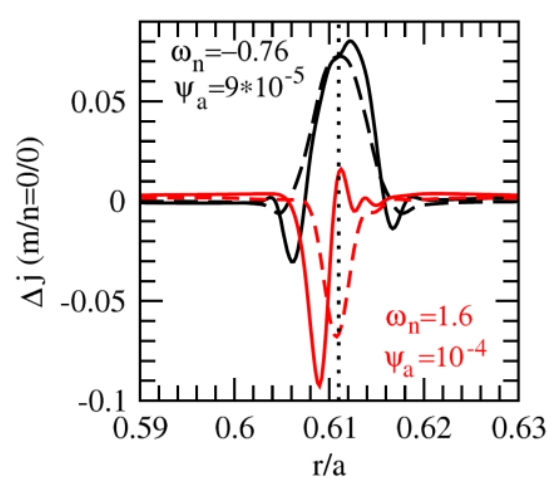

Figure $4 \quad$ Local radial profiles of $\Delta j_{0 / 0}$ (solid curve) and $\Delta j_{b, 0 / 0}$ (dashed) with $f_{b}=0.18$. The black curves are for $\omega_{n}=-0.76$ and $\psi_{a, 3 / 2}=9 \times 10^{-5}$, and the red curves are for $\omega_{n}=1.6$ and $\psi_{a, 3 / 2}=10^{-4}$. The vertical dotted line shows the $q=3 / 2$ surface location.

For the electron fluid velocity in the electron drift direction with $\omega_{\mathrm{n}}=1.6$, local radial profiles of $\Delta \mathrm{j}_{0 / 0}$ (solid curve) and $\Delta \mathrm{j}_{\mathrm{b}, 0 / 0}$ (dashed curve) are shown in figure 4 by red curves for $\mathrm{f}_{\mathrm{b}}=0.18$ and $\psi_{\mathrm{a}, 3 / 2}=10^{-4}$. In this case the RMP has not penetrated in neither, and the island width is $0.0054 \mathrm{a}$. The local radial gradient of $\mathrm{j}_{0 / 0}$ is decreased $\left(\left|j_{0 / 0}^{\prime} / j_{0 / 0}\right|\right.$ is increased) on the inner side of the $\mathrm{q}=3 / 2$ surface except in a thin layer very close to the surface, similar to that caused by a local rf current drive around the resonant surface in the electron drift direction (counter-current direction). This is known to 
increase the $\Delta^{\prime}$ value and is destabilizing for the island growth, being consistent with the results shown in figure 3. However, in this case the amplitude of $\Delta \mathrm{j}_{\mathrm{b}, 0 / 0}$ is smaller than that of $\Delta \mathrm{j}_{0 / 0}$, and their radial locations are slightly shifted, indicating the role of the nonlinearity in Ohm's law in affecting the local $j_{0 / 0}$ profile.

It is known theoretically that RMPs can increase (decrease) the local electron density of the $\mathrm{m} / \mathrm{n}=0 / 0$ component around the resonant surface for the plasma rotation in the ion (electron) drift direction [23,24], as also observed in experiments [25,27-30]. In agreement with numerical calculation results, the quasi-linear formula shows that [23]

$$
\mathrm{r}_{\mathrm{s}}\left(\Delta \mathrm{n}_{\mathrm{e}}\right)^{\prime} / \mathrm{n}_{\mathrm{e} 0}=\mathrm{d}_{1}\left(\omega *_{\mathrm{e}} 0 \mathrm{r}_{\mathrm{s}}^{2} / \mathrm{D}_{\perp}\right)\left(1-\omega_{0}\right)\left|\mathrm{b}_{1 \mathrm{r}} / \mathrm{B}_{0 \mathrm{t}}\right|^{2} / \mathrm{m}
$$

at $\mathrm{r}=\mathrm{r}_{\mathrm{s}}$ for a static RMP, where $\Delta \mathrm{n}_{\mathrm{e}}=\left(\mathrm{n}_{\mathrm{e}, 0 / 0}-\mathrm{n}_{\mathrm{e}}\right)$ is the difference between the $\mathrm{m} / \mathrm{n}=0 / 0$ component of electron density in steady state and the original equilibrium one, and $b_{1 r}$ is the local radial magnetic field perturbation. The local electron density gradient decreases $\left(\left|n_{\mathrm{e}}{ }^{\prime} / \mathrm{n}_{\mathrm{e}}\right|\right.$ increases since the equilibrium density gradient is negative) around the resonant surface for $\omega_{0}>1$ or $\omega_{n}<0$, while in the opposite limit $\left(\Delta \mathrm{n}_{\mathrm{e}}\right)^{\prime}>0$. As the bootstrap current density is proportional to the electron density gradient, equation (10) explains the change in the $\mathrm{m} / \mathrm{n}=0 / 0$ component bootstrap current density shown in figure 4.

Corresponding to figure 4 , local radial profiles of the radial electron density gradient of the 0/0 component are shown in figure 5 , The density gradient decreases $\left(\left|\mathrm{n}_{\mathrm{e}}{ }^{\prime} / \mathrm{n}_{\mathrm{e}}\right|\right.$ increases $)$ around the resonant surface for $\omega_{n}=-0.76$ but increases for $\omega_{n}=1.6$, as expected from equation (10).

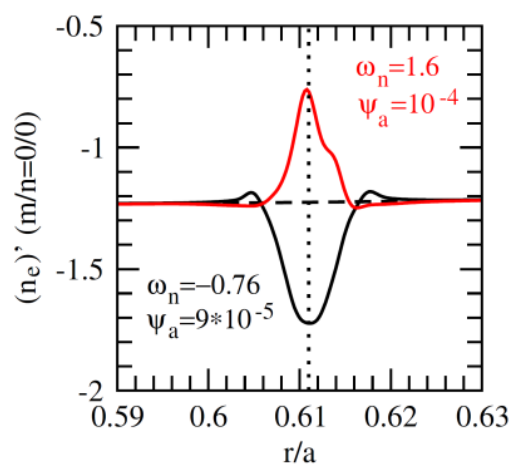

Figure $5 \quad$ Corresponding to figure 4, local radial profiles of the radial electron density gradient of the 0/0 component with $f_{b}=0.18$. The black curve is for $\omega_{n}=-0.76$ and $\psi_{a, 3 / 2}=9 \times 10^{-5}$, and the red curve is for $\omega_{n}=1.6$ and $\psi_{a, 3 / 2}=10^{-4}$. The dashed curve shows the radial gradient of the original equilibrium electron density. The vertical dotted line marks the $q=3 / 2$ surface location.

To further take into account the electron temperature perturbations, the electron heat transport equation [24], 


$$
\frac{3}{2} n_{e} \frac{d T_{e}}{d t}=d_{1} T_{e} \nabla_{||} j-T_{e} n_{e} \nabla_{\|} v_{\|}+n_{e} \nabla \cdot\left(\chi_{||} \nabla_{||} T_{e}\right)+n_{e} \nabla \cdot\left(\chi_{\perp} \nabla_{\perp} T_{e}\right)+S_{p}
$$

is solved together with equations (1)-(7), where $\chi$ the heat conductivity, and $\mathrm{S}_{\mathrm{p}}$ is the heat source. A parabolic profile for the equilibrium electron temperature is assumed, and the equilibrium electron diamagnetic drift frequency is $\omega *_{\mathrm{e} 0}=4.82 \times 10^{5} / \tau_{\mathrm{R}}\left(\mathrm{f}_{\mathrm{e}_{0} 0}=3.3 \mathrm{kHz}\right)$ at the $\mathrm{q}=3 / 2$ surface for $\mathrm{m}=3$.

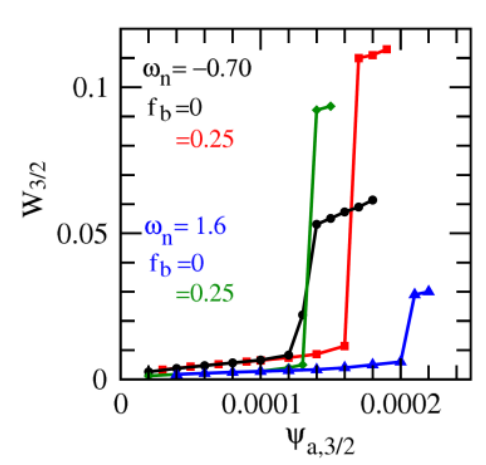

Figure $6 \quad$ The $m / n=3 / 2$ magnetic island width in steady state as a function of $\psi_{a, 3 / 2}$ for (1) $\omega_{n}=-0.70$ with $f_{b}=0$ (black curve) and 0.25 (red); (2) $\omega_{n}=1.6$ with $f_{b}=0$ (blue) and 0.25 (green).

Assuming $\chi_{\perp}=\mu$ and the ratio between the parallel and perpendicular heat conductivity to be $\chi_{\|} / \chi_{\perp}=10^{9}$, the $\mathrm{m} / \mathrm{n}=3 / 2$ island width in steady state is shown as a function of $\psi_{\mathrm{a}, 3 / 2}$ in figure 6 for $\mathrm{f}_{\mathrm{b}}=0$ and 0.25 with $\omega_{\mathrm{n}}=-0.70$ and 1.6 , respectively, keeping other input parameters unchanged. For the electron fluid velocity in the ion drift direction $\left(\omega_{n}=-0.70\right)$, the penetration threshold is larger for $f_{b}=0.25$ (red curve) than that for $f_{b}=0$ (black), indicating again the stabilizing role of the bootstrap current perturbation in this case. For the electron fluid velocity in the electron drift direction $\left(\omega_{n}=1.6\right)$, the opposite results are again obtained. The radial profiles of $\Delta \mathrm{j}_{0 / 0}$ and $\Delta \mathrm{j}_{\mathrm{b}, 0 / 0}$ are similar to that shown in figure 4, and the change in the local electron density and temperature gradient by RMPs is similar to that found before $[23,24]$. With $\mathrm{f}_{b}=0.25$, the penetration threshold is larger for $\omega_{\mathrm{n}}=-0.70$ (red) than that for $\omega_{n}=1.6$ (green), showing the asymmetry of field penetration on the two sides of $\omega_{n}=0$, as observed in experiments [31]. Comparing the red curves in figures 2 and 3 with $f_{b}=0.18$, such an asymmetry is also seen, since the penetration threshold is the same for $\omega_{\mathrm{n}}=-0.70$ and $\omega_{\mathrm{n}}=1.6$.

Due to the inclusion of the heat transport equation, the $3 / 2$ mode is linearly unstable without applying RMPs, being a micro-tearing type mode driven by the electron temperature gradient as found before [21]. The mode saturates in the small island regime in the nonlinear phase with a saturated island width around $0.01 \mathrm{a}$ for $\mathrm{f}_{\mathrm{b}}=0$ and 0.25 .

In addition to the results for the $\mathrm{m} / \mathrm{n}=3 / 2 \mathrm{NTM}$ shown above, similar results have also been found for NTMs of other mode numbers. 


\section{Discussion and summary}

In existing theory for NTMs' onset, only the helical component of bootstrap current perturbations, resulting from the (partial) flattening of plasma pressure inside a magnetic island, is taken into account [1-3]. This helical component is destabilizing for the mode onset when the electron fluid velocity is in the electron drift direction, as shown in figure 3, but it is not so important for the electron fluid velocity in the opposite direction, as shown in figure 2.

In addition to this helical component, the local $\mathrm{m} / \mathrm{n}=0 / 0$ component of the electron density gradient can also be significantly changed by RMPs or error field [23], leading to a corresponding change of the local bootstrap and plasma current density gradient. When the electron fluid velocity is in the ion drift direction, the most common case in tokamak experiments with neutral bean injection in the plasma current (ion drift) direction, this effect is stabilizing for NTMs' onset and is larger than that due to the helical bootstrap current perturbation, so that a larger bootstrap current density leads to a larger onset threshold. For the electron fluid velocity in the electron drift direction, however, NTMs are more easily to be triggered by RMPs with increasing bootstrap current density. In this case, in addition to the helical bootstrap current, the change in the $\mathrm{m} / \mathrm{n}=0 / 0$ component of local plasma current density gradient, caused by both the bootstrap current perturbation and the nonlinearity in Ohm's law, is also destabilizing.

Our results indicate that the bootstrap current term in the modified Rutherford equation, utilized to study the onset of NTMs for many years [1-3], should be revised to take into account the nonlinear two-fluid effects. The plasma current density perturbation of the $0 / 0$ component corresponds to a nonlinear modification of the $\Delta^{\prime}$ value, and it can be either stabilizing or destabilizing, depending on the electron fluid velocity. The effects studied in this paper are expected to be more important for the advanced scenario of a reactor plasma with a large fraction of bootstrap current.

In summary, the NTM's onset driven by RMPs is studied numerically based on two-fluid equations. Due to the change of the local radial profile of the bootstrap and plasma current density of the $\mathrm{m} / \mathrm{n}=0 / 0$ component, a larger onset threshold of NTMs is found for a larger bootstrap current density, when the bi-normal electron fluid velocity is in the ion drift direction. For the electron fluid velocity in the electron drift direction, the opposite results have been found. 


\section{References}

[1] Carrera, R., Hazeltine, R.D., Kotschenreuther, M. 1986 Phys. Fluids 29, 899

[2] Fitzpatrick R. 1995 Phys. Plasmas 2, 825

[3] Sauter O. et al 1997 Phys. Plasmas 41654

[4] Chang Z. et al 1995 Phys. Rev. Lett. 744663

[5] Zohm H. et al 2001 Phys. Plasmas 82009

[6] Günter S. et al 2001 Phys. Rev. Lett. 87275001

[7] La Haye R.J., Lao L.L., Strait E.J. and Taylor T.S. 1997 Nucl. Fusion 37397

[8] Gude A., Günter S. and Sesnic S. et al 1999 Nucl. Fusion 39127

[9] La Haye R. J. 2006 Phys. Plasmas 13055501

[10] Buttery R.J. et al 2008 Phys. Plasmas 15056115

[11] Igochine V. et al 2014 Phys. Plasmas 21110702

[12] Smolyakov A.I. 1993 Plasma Phys. Control. Fusion 35657

[13] Wilson H.R., Connor J.W., Hastie R.J. and Hegna C.C. 1996 Phys. Plasmas 3248

[14] Waelbroeck F.L. and Fitzpatrick R. 1997 Phys. Rev. Lett. 781703

[15] Waelbroeck F.L., Connor J.W. and Wilson H.R. 2001 Phys. Rev. Lett. 87215003

[16] Connor J.W., Waelbroeck F.L. and Wilson H.R. 2001 Phys. Plasmas 82835

[17] Fitzpatrick R., Waelbroeck F.L. and Militello F. 2006 Phys. Plasmas 13122507

[18] Glasser A.H., Greene J.M. and Johnson J.L. 1976 Phys. Fluids 19567

[19] Lütjens H, Luciani J-F and Garbet X 2001 Phys. Plasmas 84267

[20] Lütjens H, Luciani J-F and Garbet X 2001 Plasma Phys. Control. Fusion 43 A339

[21] Yu Q. 2010 Nucl. Fusion 50025014

[22] Hazeltine R. D. et al 1985 Phys Fluids 282466.

[23] Yu Q. and Günter S. 2009 Nucl. Fusion 49062001

[24] Yu Q. and Günter S. 2011 Nucl. Fusion 51073030

[25] Hender T.C. et al 1992 Nucl. Fusion 32, 2091

[26] Yu Q., Günter S. and Lackner K. 2018 Nucl. Fusion 58054003

[27] K.H. Finken, S.S. Abdullaev, M.W. Jakubowski et al., 2007 Phys. Rev. Letts, 98, 065001

[28] Hu Q. et al 2014 Nucl. Fusion 54122006

[29] Hu Q. et al, 2019 Phys. Plasmas 26120702

[30] Hu Q. et al, 2020 Nucl. Fusion, in press, https://doi.org/10.1088/1741-4326/ab8545

[31] Koslowski H.R., Liang Y., Krämer-Flecken A. et al 2006 Nucl. Fusion 46, L1 